\title{
Caracterización de ciclones casi tropicales en el Mediterráneo: un caso en el mar Balear
}

https://doi.org/10.31978/639-19-010-0.265

\author{
María Ángeles Picornell Alou1 (mpicornella@aemet.es) \\ Joan Campins Pons ${ }^{1}$ (jcampinsp@aemet.es)
}

${ }^{1}$ AEMET / Delegación Territorial en Illes Balears

\begin{abstract}
RESUMEN
En la región mediterránea ocasionalmente se han observado pequeños ciclones muy intensos que presentan características propias de ciclones tropicales, como una estructura nubosa en forma de espiral, con un «ojo» central, y un núcleo cálido en su estructura interna. Van acompañados de fuertes vientos y lluvia intensa y se les denomina medicanes (MEDI-terranean hurri-CANES) o ciclones casi tropicales. En este trabajo se presentan unos criterios para identificar este tipo de ciclones en las salidas de los modelos numéricos de predicción, lo que posibilita avanzar hacia una climatología objetiva así como a la predicción sistemática de estos fenómenos. Además, a partir del reanálisis ERA5 se estudia la evolución de un ciclón muy intenso que se desarrolló en el mar Balear en noviembre de 2011. Su evolución en el espacio de las fases revela la presencia de un núcleo cálido profundo y simétrico, por lo que el sistema se identifica como un ciclón casi tropical o medicane.
\end{abstract}

PALABRAS CLAVE: medicane; ciclón casi tropical; núcleo cálido; diagramas de fase; Mediterráneo.

\section{INTRODUCCIÓN}

La región mediterránea se ve afectada con frecuencia por ciclones extratropicales de tipología muy variada (CAmpins et al., 2011). Ocasionalmente se forman un tipo de ciclones muy intensos cuyo aspecto recuerda a los ciclones tropicales pero con un tamaño mucho menor, y reciben el nombre de medicanes (MEDI-terranean hurri-CANES) o ciclones casi tropicales. Estos ciclones se caracterizan por ir acompañados de una estructura nubosa en forma de espiral, con un «ojo» central, son poco frecuentes y se desarrollan en zonas marítimas, por lo que a menudo resultan difíciles de identificar. También se caracterizan por la presencia de un núcleo cálido profundo, asociado con la liberación de calor latente, y son térmicamente simétricos. Si bien el ciclón puede tener una vida de varios días, es posible que las características tropicales se limiten a un periodo menor, incluso solamente unas pocas horas, y durante este periodo su tamaño se reduce considerablemente, tomando un diámetro entre 100 y $300 \mathrm{~km}$ (Tous y RoMERo, 2013). Llevan asociados fuertes vientos y lluvia intensa y pueden provocar efectos adversos, especialmente cuando alcanzan zonas costeras, lo que hace muy necesaria su localización. Estos ciclones se desarrollan en entornos inicialmente baroclinos, normalmente con presencia de una dana o una vaguada en niveles altos que, al aproximarse a una zona marítima relativamente más cálida, provoca un gran desequilibrio termodinámico. Los flujos de calor, principalmente por la interacción aire-mar y por procesos convectivos, juegan un papel fundamental en la formación y mantenimiento de los medicanes (EMANUeL, 2005).

Con el fin de describir de forma adecuada estas estructuras y establecer unos criterios que permitan identificar medicanes a partir de las salidas de modelos numéricos de predicción (MNP), se ha adaptado la metodología 
para detectar y seguir la evolución de ciclones mediterráneos (PiCornell et al., 2001, CAmPins et al., 2006), así como los parámetros que definen el espacio de las fases propuestos por HART (2003), a las características de estas perturbaciones intensas de pequeña escala que se desarrollan en la región mediterránea (PiCoRnell et al., 2014).

En noviembre de 2011 un medicane afectó al mar Balear. Se formó en el golfo de León, siguió un recorrido marítimo casi circular, acompañado de intensa actividad convectiva, y finalmente se intensificó y adquirió características tropicales. Se registraron fuertes vientos y se recogió gran cantidad de lluvia que provocó inundaciones en el sur de Francia. En este trabajo se estudia su evolución utilizando las citadas herramientas a partir del reanálisis ERA5 (HeRsBACH y DeE, 2016).

La estructura de este trabajo es la siguiente: en la sección 2 se presentan las herramientas utilizadas para describir ciclones mediterráneos; en la sección 3 esta metodología se adapta para describir el tipo de ciclón objeto de este estudio, los medicanes. En el apartado 4, se describe la evolución de un medicane que afectó al mar Balear en noviembre de 2011. Las herramientas descritas permiten avanzar hacia una climatología de medicanes y hacia la predicción de los mismos, como se explica en la sección 5. Finalmente, en el último apartado se presentan las conclusiones de este trabajo.

\section{HERRAMIENTAS PARA DESCRIBIR CICLONES MEDITERRÁNEOS}

\subsection{Detección y seguimiento de ciclones}

En el procedimiento original desarrollado en AEMET para la detección y seguimiento de los ciclones mediterráneos (Picornell et al., 2001, CAMPINs et al., 2006), se define un ciclón como un mínimo del campo de presión a nivel del mar (MSLP) que supera un umbral de gradiente de presión $(\nabla p \geq 0,5 \mathrm{hPa} / 100 \mathrm{~km})$ en 6 de las 8 direcciones principales alrededor del mínimo. Se considera como dominio del ciclón el área encerrada por la línea de vorticidad nula. Para determinar la trayectoria del ciclón se parte de la hipótesis de que el viento en $700 \mathrm{hPa}$ es el que dirige su movimiento. Como medida de la intensidad del ciclón tomamos la circulación geostrófica media o el gradiente medio de MSLP. Este criterio de selección es poco restrictivo y permite detectar desde centros ciclónicos poco intensos a ciclones profundos. El tamaño típico de estos ciclones oscila entre los 400 y los 600 km de diámetro.

\subsection{Espacio de las fases de los ciclones}

El espacio de las fases de los ciclones, EFC (cyclone phase space, CPS, en inglés), resulta muy útil para clasificar los ciclones teniendo en cuenta su estructura térmica tridimensional (HART, 2003). Las posiciones que ocupa un ciclón en este espacio informan de su tipología y de su evolución. Hart propone tres parámetros para construir este espacio. El parámetro B, que mide el gradiente de la temperatura media del estrato, es una medida de la simetría térmica del ciclón y permite distinguir entre un ciclón simétrico/no frontal de uno asimétrico/frontal. Los otros dos parámetros, el viento térmico en la troposfera baja, $-V_{L}^{T}$, y el viento térmico en la troposfera alta, $-V_{U}^{T}$, permiten distinguir entre una estructura de núcleo frío frente a una de núcleo cálido.

Hart recomienda calcular estos parámetros en un círculo que se adapte al tamaño de la perturbación. Con este fin utilizamos el radio del núcleo cálido en el nivel intermedio para calcular el parámetro B y el viento térmico. En caso de ausencia de núcleo cálido tomamos un valor por defecto. Estos valores son mucho menores a los $500 \mathrm{~km}$ de radio que utiliza Hart, ya que en su caso pretende describir ciclones tropicales, de tamaño mucho mayor. Por otra parte, el cálculo de estos parámetros se basa en el análisis de dos capas troposféricas. Teniendo en cuenta que la troposfera en el Mediterráneo presenta una extensión vertical menor que en los trópicos, dividimos la troposfera en dos capas mediante los niveles 400-700-925 hPa en lugar de los niveles utilizados por Hart, 300-600-900 hPa (PiCORNELl et al., 2014). 


\section{DEFINICIÓN DE MEDICANE}

Mediante estas herramientas proponemos una definición de medicane a partir de los campos de MNP. Exigimos tres requisitos para que un ciclón sea considerado medicane: debe ser un ciclón muy intenso $(\nabla p \geq 3,2 \mathrm{hPa} / 100 \mathrm{~km})$, simétrico $(\mathrm{B}<10 \mathrm{~m})$ y debe presentar un núcleo cálido profundo $\left(-V_{L}^{T}>0,-V_{U}^{T}>0\right)$. Esta definición debe completarse con información proveniente de otras fuentes de observación, principalmente información satelital. Su estructura nubosa se caracteriza por tener estructuras convectivas organizadas alrededor de un «ojo» central sin nubes. Para definir un medicane también se pueden tener en cuenta otros parámetros, como un umbral de viento o su tamaño. Esta definición ha sido validada con cuatro casos históricos bien documentados en la literatura, a partir de unas simulaciones que se hicieron utilizando el modelo del Centro Europeo ECMWF T1279L91Cy36r1 (ECMWF T1279), con una resolución horizontal de unos $15 \mathrm{~km}$. Los resultados de este trabajo están recogidos en PiCornell et al., 2014.

\section{MEDICANE 6-9 NOVIEMBRE 2011}

\subsection{Descripción del episodio}

Nos centramos en el caso de un ciclón de características tropicales, el medicane Rolf, que afectó al Mediterráneo occidental del 6 al 9 de noviembre de 2011. Analizaremos su evolución a partir de la información satelital y a partir de algunos campos del reanálisis ERA5. Este reanálisis, que se está preparando actualmente en el Centro Europeo, utiliza una versión avanzada del modelo IFS del ECMWF, IFS Cycle 41r2, y ofrece una resolución horizontal de $31 \mathrm{~km}$ y 137 niveles en la vertical. Proporciona una estimación horaria de los campos meteorológicos y utiliza más observaciones de satélite en la asimilación de datos que reanálisis anteriores.

El día 5 de noviembre, en el Mediterráneo occidental, se localiza una zona de bajas presiones al oeste de Cerdeña, con una estructura térmica frontal. A niveles altos una dana se aproxima a la zona y favorece la profundización de un centro ciclónico en el golfo de León. Este ciclón se mueve siguiendo una trayectoria casi circular, como muestra la fig. 4b. En una primera fase, durante el día 6, empieza a organizarse convección en la zona, se forma un núcleo cálido a nivel bajo en el centro del ciclón, reflejado en el campo de temperatura a $850 \mathrm{hPa}$, que se desplaza hacia el sur y afecta al archipiélago balear, donde se registran gran cantidad de lluvia y vientos fuertes. A lo largo del día 7 el ciclón se mueve sobre el mar hacia el norte, intensificándose y expandiéndose el núcleo cálido a niveles superiores (ya puede apreciarse en el campo de temperatura a $500 \mathrm{hPa}$ ). En la imagen de satélite de este día, mostrada en la figura 1a, se observan ya bandas convectivas intensas que se enroscan alrededor del centro ciclónico y siguiendo su desplazamiento hacia el norte a lo largo del día 8.

A primeras horas del día 8 el ciclón alcanza su máxima intensidad, con una presión central estimada de $991 \mathrm{hPa}$ y vientos estimados desde ASCAT METOP-A de $45 \mathrm{kt}$, mostrados en la figura 2. De hecho la imagen del día 8 a mediodía (fig. 1b) ya muestra una estructura nubosa convectiva circular y un «ojo» central libre de nubes, estructura propia de un medicane. Los campos analizados para el día 8 a las 00 UTC (fig. 3) muestran un mínimo de presión en superficie muy intenso, con notable gradiente de presión, y un núcleo cálido en la vertical, tanto a 850 como a $500 \mathrm{hPa}$. Todo ello confirma que el día 8 , alrededor de las $00 \mathrm{UTC}$, tiene lugar la transición de ciclón extratropical a ciclón casi tropical. Finalmente, al día siguiente el ciclón se desplaza hacia el sur de Francia, como muestra la imagen del día 9 (fig. 1c) y se debilita al tocar tierra.

Si bien la evolución de la presión en el centro del ciclón no queda bien representada en el reanálisis (la caída de presión se estimó mucho mayor, alcanzando un valor mínimo de $991 \mathrm{hPa}$ ), el gradiente de presión analizado sí que refleja el momento de mayor intensidad (fig. 4a). 

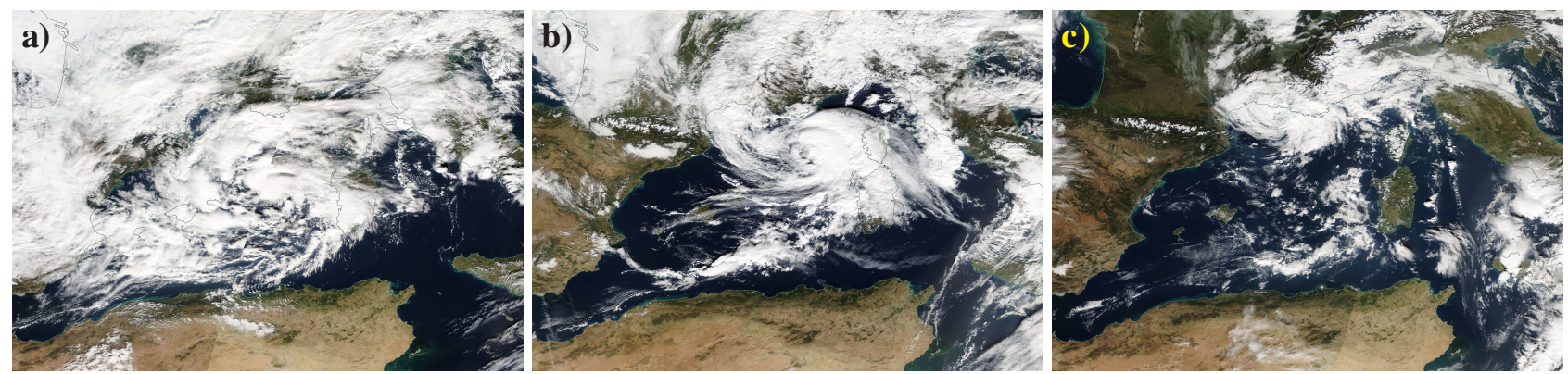

Figura 1. Imágenes del sensor MODIS del satélite Aqua de la NASA correspondientes a los días 7 (a), 8 (b) y 9 (c) de noviembre.

a)

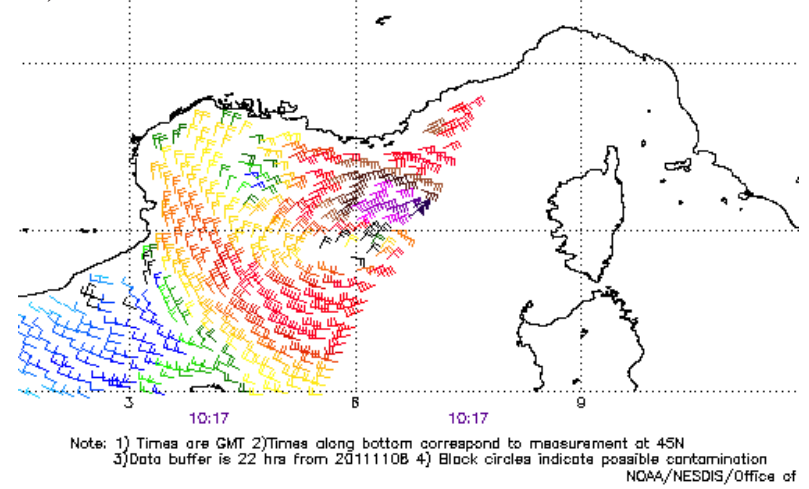

b)

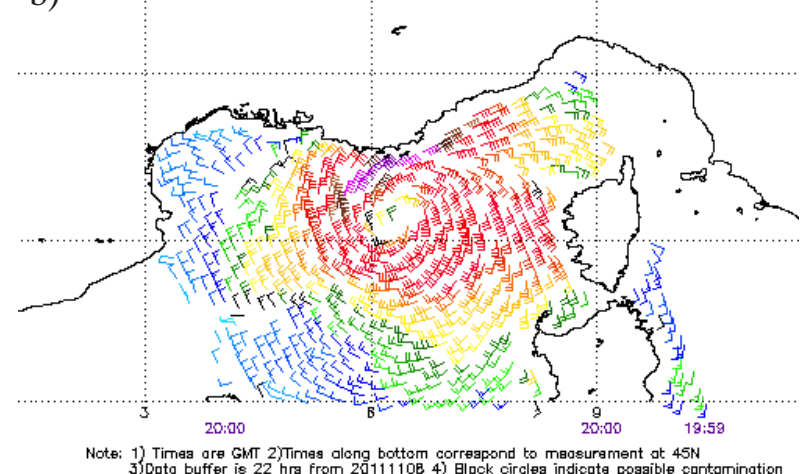

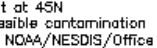

Figura 2. Datos de viento obtenidos de ASCAT METOP-A correspondientes al día 8 de noviembre a las 10:17 UTC (a) y a las 20:00 UTC (b).

a)

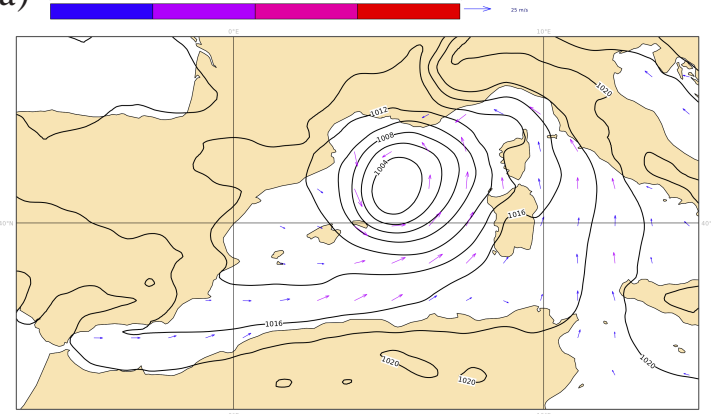

b)

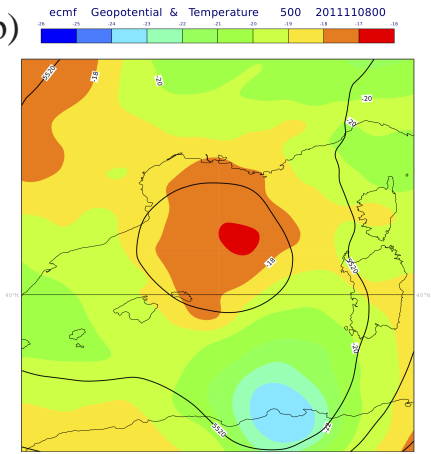

c)

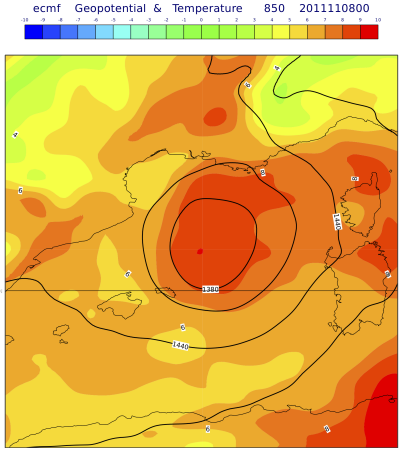

Figura 3. Análisis del ERA5 de a) MSLP, b) altura geopotencial y temperatura a 500 y c) a $850 \mathrm{hPa}$ del día 8 de noviembre de 2011 a las 00 UTC, momento de la transición tropical.

a)

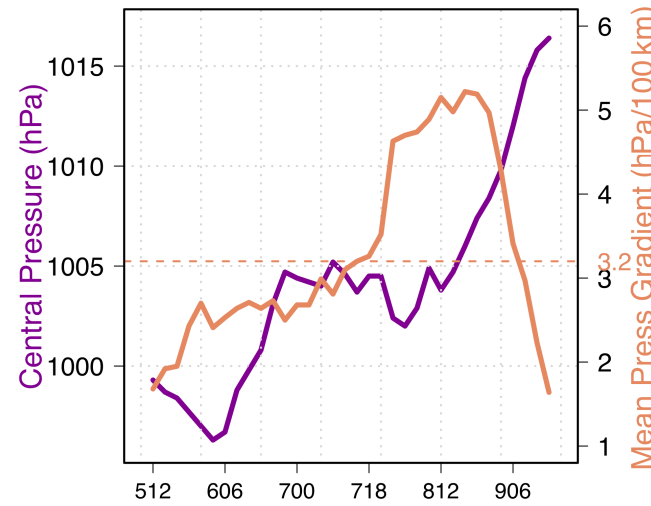

b)

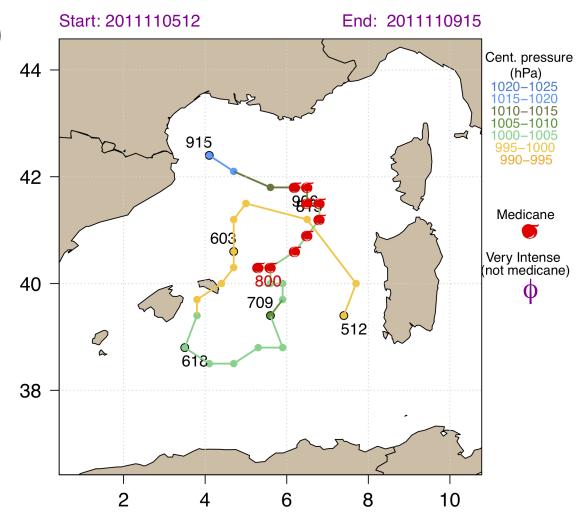

Figura 4. a) Evolución de la presión en el centro y del gradiente de presión y b) trayectoria del ciclón. El símbolo $\sigma$ indica el momento en que es medicane. 
a)

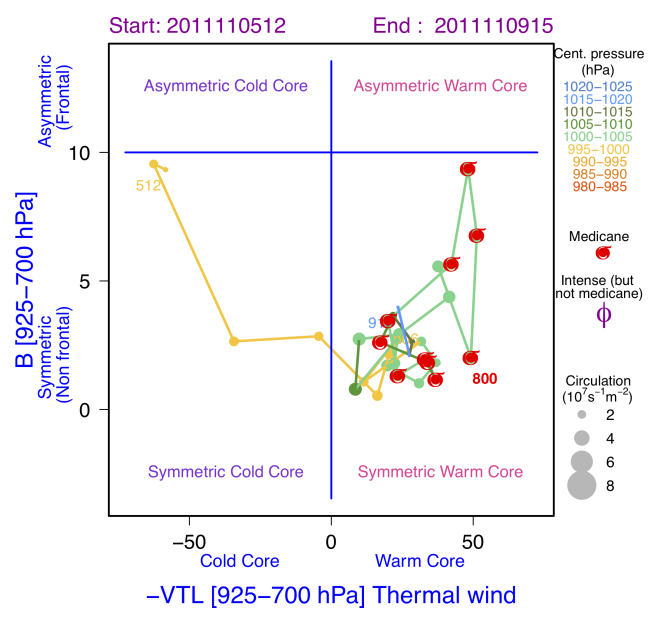

b)

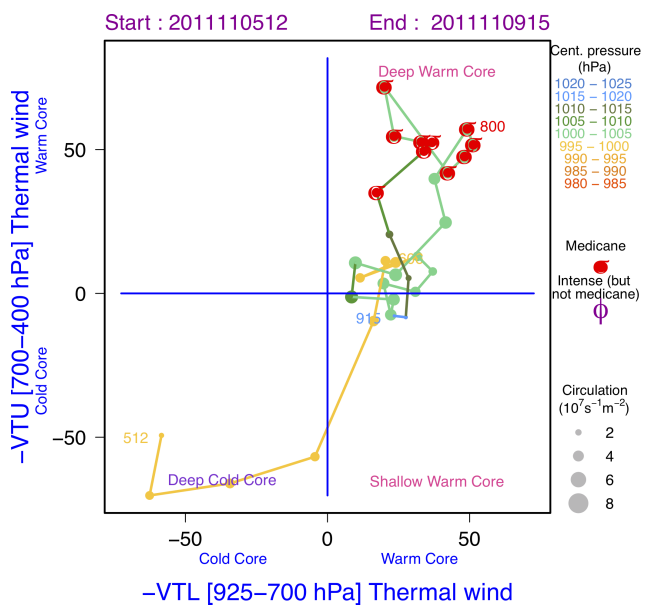

Figura 5. Diagramas de fase. El símbolo

La evolución del ciclón queda reflejada en los diagramas de las fases (fig. 5). Se trata de un sistema simétrico, que empieza siendo un ciclón con núcleo frío pero se forma un núcleo cálido, inicialmente en la troposfera media-baja y que se hace profundo el día 7. El día 8 a las 00 UTC el ciclón se hace muy intenso y cumple los requisitos exigidos para considerarlo como un medicane. Estas características se mantienen hasta el día 9 a las 03 UTC, cuando el ciclón se aproxima a tierra y pierde intensidad.

\section{HACIA UNA CLIMATOLOGÍA Y UNA PREDICCIÓN DE MEDICANES}

Con el reanálisis ERA5 se dispondrá en breve de una base de análisis homogénea desde 1979 hasta la actualidad. Este reanálisis ofrece la posibilidad de describir los medicanes de las últimas cuatro décadas a partir de un mismo modelo, lo que permite avanzar hacia una definición objetiva de un medicane (todavía en proceso) y hacia una climatología de este tipo de ciclones. Aplicando el procedimiento de detección al periodo completo, podremos conocer mejor algunas de sus características, como la frecuencia con la que ocurren estos fenómenos, las zonas y las épocas en las que preferentemente se forman e intensifican y el momento de su transición tropical. De momento hemos analizado algunos casos, entre ellos el medicane Rolf, ocurridos entre 2008 y 2017, periodo del que ya está disponible el reanálisis en estos momentos, utilizando las herramientas descritas en el apartado anterior. Las trayectorias de estos casos se presentan en la figura 6. También se incluyen en esta figura las trayectorias de los cuatro medicanes ocurridos entre 1982 y 1996 , estudiados a partir de las simulaciones con el ECMWF T1279 para validar la definición de medicane. Estos primeros resultados confirman los obtenidos en estudios anteriores, como en MiglieTTA et al., 2013, que indican que los medicanes ocurren preferentemente en otoño e invierno y unos pocos en primavera, y se localizan principalmente en el Mediterráneo central (mar Jónico) y occidental.

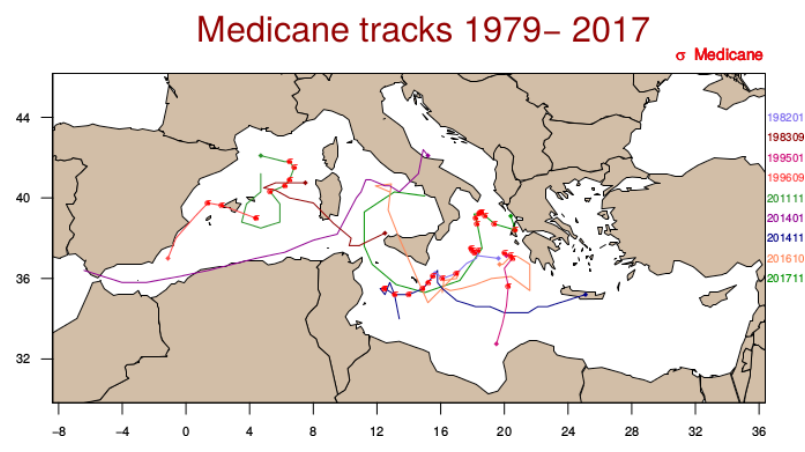

Figura 6. Trayectorias de algunos medicanes, obtenidas con el ECMWF T1279 (1982-1996) y con el reanálisis ERA5 (2011-2017).
Con estas herramientas y un modelo de predicción de suficiente resolución para representar estas estructuras también podemos avanzar en el camino de la predicción de estos fenómenos. Algunos modelos deterministas, como el de alta resolución del Centro Europeo, HRES-IFS o ECHRES (con una resolución horizontal de $9 \mathrm{~km}$ y 137 niveles verticales), y el Harmonie-Arome (con 2,5 km de resolución horizontal y 65 niveles verticales) pueden resultar adecuados. Dada la severidad, el pequeño tamaño y la poca frecuencia con la que aparecen los medicanes, algunos modelos probabilistas o sistemas de predicción por conjuntos (SPC), 
como el del Centro Europeo, el ECENS o ENS-IFSECEPS (con 51 miembros con 16 km de resolución horizontal), y especialmente el AEMET- $\gamma$ SREPS (con 20 miembros de alta resolución a 2,5km, no hidrostáticos), serán sin duda muy útiles para la predicción de este fenómeno. Estos sistemas informan de los escenarios más probables (en los que coinciden el mayor número de miembros), pero también permiten que algunos miembros (aunque sean pocos) alerten de la posibilidad de ocurrencia de estos fenómenos severos. En estos momentos están en desarrollo algunos productos para presentar de manera concisa toda esta información obtenida de los SPC (Picornell y Campins, 2018).

\section{CONCLUSIONES}

En el Mediterráneo se han observado pequeños ciclones de tipo tropical, llamados medicanes. Se producen con muy baja frecuencia pero sus efectos son devastadores en algunos casos. Se desarrollan en zonas marítimas, lo que dificulta su identificación. Presentamos una definición de «posible» medicane que permite su detección y caracterización a partir de modelos numéricos, que debe complementarse con información de otras fuentes.

Un ciclón muy intenso afectó al Mediterráneo occidental del 6 al 9 de noviembre de 2011. A partir del reanálisis ERA5 se caracteriza como un ciclón muy intenso con una estructura térmica cálida y simétrica. Este ciclón lleva asociada una estructura nubosa en forma de espiral con un «ojo» central y se registraron rachas fuertes de viento y caída de presión. Se confirma su carácter tropical y se identifica como medicane.

En este trabajo se presenta una herramienta para detectar y caracterizar medicanes a partir de modelos numéricos. Por una parte, posibilita avanzar hacia una climatología de medicanes a partir de un reanálisis de cierta resolución, como el ERA5, que a su vez contribuye a una definición definitiva de estos fenómenos. Por otra, facilita la predicción de medicanes a partir de modelos deterministas de alta resolución y a partir de modelos probabilistas, como el ECENS o incluso de alta resolución como el AEMET- $\gamma$ SREPS. La predicción probabilista de alta resolución resulta muy adecuada para alertar de estos fenómenos severos que de vez en cuando afectan al Mediterráneo y a sus zonas costeras.

\section{REFERENCIAS}

CAmpins, J., JansÀ, A. y Genovés, A., 2006. Three-dimensional structure of western Mediterranean cyclones. Int. J. Climatol., 26 (3), 323-343.

Campins, J., Jansà, A., Genovés, A. y Picornell, M. A., 2011. Climatology of Mediterranean cyclones using the ERA-40 dataset. Int. J. Climatol., 31, 1596-1614.

EMANUEL, K. A., 2005. Genesis and maintenance of «Mediterranean hurricanes». Adv. Geosci., 2, 217-220. http://dx.doi.org/10.5194/adgeo-2-217-2005.

HART, R. E., 2003. A cyclone phase space derived from thermal wind and thermal asymmetry. Mon. Wea. Rev., 131, 585-616.

Hersbach, H. y DeE, D., 2016. ERA5 reanalysis is in production. ECMWF Newsletter, No. 147, ECMWF, Reading, United Kingdom, 7. www.ecmwf.int/sites/default/files/elibrary/2016/16299-newsletter-no147-spring2016.pdf.

Miglietta, M. M., Laviola, S., Malvadi, A., Conte, D., Levizzani, V., y Price, C., 2013. Analysis of tropical-like cyclones over the Mediterranean Sea through a combined modeling and satellite approach. Geophys. Res. Lett., 40, 2400-2405. 
Picornell, M. A., Jansì, A., Genovés, A. y CAmpins, J., 2001. Automated database of mesocyclones from the HIRLAM(INM) 0.5 analyses in the Western Mediterranean. Int. J. Climatol., 21, 335-354.

Picornell, M. A., CAmpins, J. y Jansà, A., 2014. Detection and thermal description of medicanes from numerical simulation. Nat. Hazards Earth Syst. Sci., 14, 1059-1070.

Picornell, M. A. y Campins, J., 2018. Predicción de medicanes. En Santos, C. (ed.): La física del caos en la predicción meteorológica, 549-559. AEMET.

Tous, M. y Romero, R., 2013. Meteorological environments associated with medicane development. Int. J. Climatol., 33, 1-14. 
